\title{
Quick and Easy Binary to dB Conversion
}

\author{
George Weistroffer \\ gweistro@yahoo.com
}

\author{
Jeremy A. Cooper \\ cooperja@gmail.com \\ Virginia Commonwealth University
}

\author{
Jerry H. Tucker \\ jhtucker@vcu.edu
}

\begin{abstract}
An algorithm is developed for converting binary integers to decibels $(\mathrm{dB})$. Compared to known alternative methods the algorithm is not only faster, but requires less memory. It also has the advantage of being easy to implement. The presented technique is useful for applications such as converting the output of an analog to digital converter to discrete $d B$ values. The speed of the algorithm is due to the fact that no floating-point operations are required. In fact, the only real time arithmetic employed is one integer subtraction. The algorithm can easily be implemented in a microcontroller or FPGA.
\end{abstract}

\section{Introduction}

A frequent task performed by embedded systems is the real time collection of analog data. One such application is the collection of acoustic data using an array of MEMS microphones [7-9]. To monitor this process, it is desirable to provide an indication in decibels $(\mathrm{dB})$ of the amplitudes of the analog inputs. In response to this need, a simple and fast algorithm has been developed for converting a binary word, representing an analog voltage, to a $\mathrm{dB}$ value.

The calculation of decibels is challenging because it involves obtaining a logarithm. Numerous methods have been proposed for calculating logarithms [1-6]. Any of these procedures could be used to first obtain the necessary logarithm and then use this logarithm to calculate the $\mathrm{dB}$ value. However, such an approach introduces unnecessary complexity and requires excessive computation time. An algorithm is presented that avoids these difficulties by directly obtaining the $\mathrm{dB}$ value. The method does not require the calculation of logarithms in real time, but consists of a two-table lookup process using very small tables. The assumption is made, as is the usual case, that a high degree of precision is not required. The effectiveness of the algorithm is demonstrated by the fact that the $\mathrm{dB}$ value of a 16-bit word can be obtained to within about $1 / 2$ $\mathrm{dB}$ using two lookup tables each containing only 16-bytes. The processor overhead in performing this conversion is minimal because the $\mathrm{dB}$ conversion simply consists of shifting the input word to obtain the indexes into two tables, and then subtracting the two values read from the tables.

\section{The Method}

Let $w$ be the value in an unsigned $n$-bit binary word, and let $w_{\mathrm{i}}$ be the $i^{\text {th }}$ bit of that word such that

$w=\left(w_{n-1} w_{n-2} \ldots w_{i} \ldots w_{0}\right)_{2}$

The binary word $w$ is assumed to represent an analog to digital converter (ADC) voltage. Let $G$ be the comparison in $\mathrm{dB}$ of $w$ to the reference of $2^{n}-1$, which is the maximum possible value of $w$. Then $G$ is given by

$G=20 \log _{10}\left(\frac{w}{2^{n}-1}\right)$

This can be rewritten as

$G=20 \log _{10}(w)-20 \log _{10}\left(2^{n}-1\right)$

Considering the computation of equation (3), it is apparent that for any particular value of $n$, the term $20 \log _{10}\left(2^{n}-1\right)$ is a constant and does not have to be computed in real time. The values of $20 \log _{10}\left(2^{n}-1\right)$ for $n$ from 8 to 20 are shown in Table 1.

Table 1 . The $G_{\mathrm{c}}$ table showing the value of $20 \log _{10}\left(2^{n}-1\right)$ for $n$ from 8 to 20 .

\begin{tabular}{|c|c|}
\hline$n$ & $20 \log _{10}\left(2^{n}-1\right)$ \\
\hline 8 & 48.1 \\
\hline 9 & 54.2 \\
\hline 10 & 60.2 \\
\hline 11 & 66.2 \\
\hline 12 & 72.2 \\
\hline 13 & 78.3 \\
\hline 14 & 84.3 \\
\hline 15 & 90.3 \\
\hline 16 & 96.3 \\
\hline 17 & 102.4 \\
\hline 18 & 108.4 \\
\hline 19 & 114.4 \\
\hline 20 & 120.4 \\
\hline
\end{tabular}


The difficulty in evaluating equation (3) is the computation of the $20 \log _{10}(w)$ term. Since $w$ changes in real time, $20 \log _{10}(w)$ must be continually recalculated. Therefore, a fast method of calculating $20 \log _{10}(w)$ is necessary. Developing such a method is made easier by recognizing that $\mathrm{dB}$ values typically do not need to be represented with high precision. Therefore, it is possible to use an approximation of $w$. To approximate $w$, only the most significant bits of $w$ will be considered.

Assuming $w \neq 0, w$ can be expressed as

$w=\left(1 w_{m-1} w_{m-2} \ldots w_{0}\right)_{2}$

where bit $m$ with $m<n$ is the left-most (most significant) " 1 " of $w$. The approximation of $w$ includes only the first $r$ bits to the right of the most significant " 1 " of $w$. So that when $m>r$

$w \geq\left(1 w_{m-1} w_{m-2} \ldots w_{m-r} 0 \ldots 0\right)_{2}$

and

$w \leq\left(1 w_{m-1} w_{m-2} \ldots w_{m-r} 1 \ldots 1\right)_{2}$

Note that when $m \leq r$ the above approximation for $w$ is equal to $w$ since all significant bits of $w$ are included.

Let $R$ be the value of $w_{m-1} w_{m-2} \ldots w_{m-r}$ so that

$R=\left(w_{m-1} w_{m-2} \cdots w_{m-r}\right)_{2}$

Then equation (5) can be rewritten as

$w \geq 2^{m}+R \times 2^{m-r}$

and equation (6) can be rewritten as

$w \leq 2^{m}+R \times 2^{m-r}+2^{m-r}-1=2^{m}+(R+1) \times 2^{m-r}-1$

From equation (8)

$$
\begin{aligned}
\log _{10}(w) & \geq \log _{10}\left(2^{m}+R \times 2^{m-r}\right) \\
& =\log _{10}\left(\left(1+R \times 2^{-r}\right) 2^{m}\right) \\
& =\log _{10}\left(2^{m}\right)+\log _{10}\left(1+R \times 2^{-r}\right) \\
& =\log _{10}(2) \log _{2}\left(2^{m}\right)+\log _{10}\left(1+R \times 2^{-r}\right) \\
& =\log _{10}(2) m+\log _{10}\left(1+R \times 2^{-r}\right)
\end{aligned}
$$

And from equation (9)

$$
\begin{aligned}
& \log _{10}(w) \leq \log _{10}\left(2^{m}+R \times 2^{m-r}+2^{m-r}-1\right) \\
& =\log _{10}\left(\left(1+R \times 2^{-r}+2^{-r}-2^{-m}\right) 2^{m}\right) \\
& =\log _{10}\left(2^{m}\right)+\log _{10}\left(1+(R+1) \times 2^{-r}-2^{-m}\right) \\
& =\log _{10}(2) \log _{2}\left(2^{m}\right)+\log _{10}\left(1+(R+1) \times 2^{-r}-2^{-m}\right) \\
& =\log _{10}(2) m+\log _{10}\left(1-2^{-m}+(R+1) \times 2^{-r}\right)
\end{aligned}
$$

The minimum value of $G, G_{\min }$, is obtained from equation (3) and equation (10).

$$
\begin{aligned}
G_{\min }= & 20 \log _{10}(2) m+20 \log _{10}\left(1+R \times 2^{-r}\right) \\
& -20 \log _{10}\left(2^{n}-1\right)
\end{aligned}
$$

The maximum value of $G, G_{\max }$, is obtained from equation (3) and equation (11).

$$
\begin{aligned}
G_{\max }= & 20 \log _{10}(2) m+20 \log _{10}\left(1-2^{-m}+(R+1) \times 2^{-r}\right) \\
& -20 \log _{10}\left(2^{n}-1\right)
\end{aligned}
$$

After eliminating the common term in equation (12) and equation (13), the difference between $G_{\max }$ and $G_{\min }$ is seen to be

$$
\begin{aligned}
G_{\max }-G_{\min }= & 20 \log _{10}\left(1-2^{-m}+(R+1) \times 2^{-r}\right) \\
& -20 \log _{10}\left(1+R \times 2^{-r}\right)
\end{aligned}
$$

But equation (14) can be written as

$$
\begin{aligned}
G_{\max }-G_{\min } & =20 \log _{10}\left(\frac{1-2^{-m}+(R+1) \times 2^{-r}}{1+R \times 2^{-r}}\right) \\
& =20 \log _{10}\left(\frac{1+R \times 2^{-r}-2^{-m}+2^{-r}}{1+R \times 2^{-r}}\right) \\
& =20 \log _{10}\left(1+\frac{2^{-r}-2^{-m}}{1+R \times 2^{-r}}\right) \\
& =20 \log _{10}\left(1+\frac{1-2^{-(m-r)}}{2^{r}+R}\right)
\end{aligned}
$$

Equation (15) is valid for $m>r$. When $m \leq r$, the approximation for $w$ is the actual value of $w$, and $G_{\max }-G_{\min }=0$.

It would be reasonable to approximate $G$ as the average of $G_{\min }$ and $G_{\max }$. This is obtained by setting the approximation of $G$ to $G_{\min }$ with an error reduction factor obtained by taking one half of $G_{\max }-G_{\min }$, as shown in equation (16). 


$$
G \approx G_{\min }+\frac{1}{2}\left(G_{\max }-G_{\min }\right)
$$

The problem with this approach is that $G_{\text {max }}-G_{\text {min }}$ is a function of both $m$ and $R$, and this makes it impossible to establish a simple two-table lookup scheme. Although it appears possible to develop an error reduction factor that avoids this problem, only the simple case of using $G_{\min }$ as an approximation for $G$ will be considered. Using this approach, the maximum error for any particular $R$ and any particular $m>0$ is the difference between $G_{\max }$ and $G_{\min }$ given by equation (15). For $m \leq 0$, the error is 0 .

For any particular value of $m$, equation (15) takes on the maximum value when $R=0$. Therefore,

$$
G_{\max }-G_{\min } \leq 20 \log _{10}\left(1+\frac{1-2^{-(m-r)}}{2^{r}}\right)
$$

Recall that for any particular application, $n$ and $r$ are fixed. It is only $m$ and $R$ that vary in real time. Separating equation (12) into the part that depends on $m$, the part that depends on $R$, and the constant part that depends on neither $m$ nor $R$ gives

$$
G_{\min }=G_{m}(m)+G_{R}(R, r)-G_{c}(n)
$$

Using this as the approximation for $G$ gives

$$
G \approx G_{m}(m)+G_{R}(R, r)-G_{c}(n)
$$

Where

$$
G_{m}(m)=20 \log _{10}(2) m
$$

$G_{R}(R, r)$ in real time is effected only by $R$ and is given by

$$
G_{R}(R, r)=20 \log _{10}\left(1+R \times 2^{-r}\right)
$$

$G_{c}(n)$ does not vary in real time, and is given by

$$
G_{c}(n)=20 \log _{10}\left(2^{n}-1\right)
$$

Equation (17) predicts the maximum error and leads to the conclusion that

max error $\leq 20 \log _{10}\left(1+\frac{1-2^{-(m-r)}}{2^{r}}\right)$ when $m>r$

and

max error $=0$ when $m \leq r$
Since $m<n$, equation (23) takes on its maximum value when $m=n-1$. Therefore, the worst-case error is given by

$$
\text { worst case error }=20 \log _{10}\left(1+\frac{1-2^{-(n-1-r)}}{2^{r}}\right)
$$

This worst-case error based on equation (25) is shown in Table 2.

Table 2. The worst-case error in $\mathrm{dB}$ as a function of $n$ and $r$.

\begin{tabular}{c|c|c|c|c|c|}
$n$ & $r=1$ & $r=2$ & $r=3$ & $r=4$ & $r=5$ \\
\cline { 2 - 6 } 8 & 3.48 & 1.88 & .96 & .46 & .2 \\
\cline { 2 - 6 } 9 & 3.50 & 1.91 & .99 & .49 & .23 \\
\cline { 2 - 6 } 10 & 3.51 & 1.92 & 1.01 & .51 & .25 \\
\cline { 2 - 6 } 11 & 3.52 & 1.93 & 1.02 & .52 & .26 \\
\hline 12 & 3.52 & 1.93 & 1.02 & .52 & .26 \\
\cline { 2 - 6 } 13 & 3.52 & 1.94 & 1.02 & .52 & .27 \\
\cline { 2 - 6 } 14 & 3.52 & 1.94 & 1.02 & .53 & .27 \\
15 & 3.52 & 1.94 & 1.02 & .53 & .27 \\
\cline { 2 - 6 } 16 & 3.52 & 1.94 & 1.02 & .53 & .27 \\
\cline { 2 - 6 } 17 & 3.52 & 1.94 & 1.02 & .53 & .27 \\
18 & 3.52 & 1.94 & 1.02 & .53 & .27 \\
\cline { 2 - 6 } 19 & 3.52 & 1.94 & 1.02 & .53 & .27 \\
\cline { 2 - 6 } 20 & 3.52 & 1.94 & 1.02 & .53 & .27 \\
\cline { 2 - 6 }
\end{tabular}

\section{Implementation}

It is apparent from equation (18) that $G$ can be calculated using two lookup tables: one for $G_{m}$ and another for $G_{R}$. The $G_{m}$-table contains $n$-entries and uses $m$ as the index into the table. The $G_{R}$-table contains $2^{r}$-entries and uses $R$ as the index into the table. The basic procedure for finding $G$ based on equation (19) is summarized by Algorithm 1.

\section{Algorithm 1. The basic algorithm.}

To convert $w$ to $\mathrm{dB}$, perform the following steps.

Initial Steps:

1. Set up the $G_{m}$ and $G_{R}$ tables.

2. Calculate $G_{c}$.

Real time steps:

1. From $w$ find $m$ and $R$.

2. Using $m$ as the index, look up the value of $G_{m}$.

3: Using $R$ as the index, look up the value of $G_{R}$.

4: Sum $G_{m}$ and $G_{R}$, and subtract the constant $G_{c}$.

In practice, it is recommended that several refinements be made to the basic algorithm. First, it is not necessary to subtract $G_{\mathrm{c}}$ each time a $\mathrm{dB}$ value is computed. It would be 
slightly more efficient to combine the $G_{c}$ value with each entry in one of the tables, such as $G_{m}$, and look up the combined value. Another refinement that can be made, to avoid having to deal with fractional values, is to scale the entries in the tables so that the integer values in the tables represent fractions of $\mathrm{dB}$, such as $1 / 2$ or $1 / 4 \mathrm{~dB}$. A final useful refinement follows from the fact that $G$ will always be negative. To avoid having to deal with negative numbers, the negation of $G$ can be represented as an unsigned integer.

The above observations lead to the conclusion that in practice it is better to compute $-k G$, where $k$ is a scale factor and $G$ is given by equation (19). $-k G$ is expressed in the form

$-k G \approx k\left(G_{c}-G_{m}\right)-k G_{R}$

The actual procedure for obtaining the $\mathrm{dB}$ value is based on the above equation and consists of setting up two tables: one for $k\left(G_{c}-G_{m}\right)$ and one for $k G_{R}$. The procedure is outlined in Algorithm 2.

Algorithm 2. The refined algorithm.

To convert $w$ to $\mathrm{dB}$, perform the following steps.

Initial step:

1. Set up tables for $k\left(G_{C}-G_{m}\right)$ and $k G_{R}$.

Real time steps:

1 . From $w$, find $m$ and $R$.

2. Using $m$ as the index, look up the value of $k\left(G_{c}-G_{m}\right)$.

3. Using $R$ as the index, look up the value of $k G_{R}$.

4. Subtract $k G_{R}$ from $k\left(G_{c}-G_{m}\right)$ to produce the negative of the scaled value of $G$.

\section{An Example Implementation}

The refined algorithm can be illustrated by presenting a specific example. Let $w$ be a 16-bit unsigned word so that $n=16$. Assume it is desired to find $G$ with a resolution of $1 / 2 \mathrm{~dB}$. Since Table 2 shows that $r=4$ will result in a resolution of about $1 / 2 \mathrm{~dB}$, let $r=4$. To use integers to represent a precision of $1 / 2 \mathrm{~dB}$, let the scale factor $k=2$. In this case, equation (26) can be written as

$-2 G \approx 2\left(G_{c}-G_{m}\right)-2 G_{R}$

Where $G_{\mathrm{c}}$ is given by Table 1 where $n=16$ as

$G_{c}(16)=20 \log _{10}\left(2^{16}-1\right)=96.3 \mathrm{~dB}$
$G_{\mathrm{m}}$ is given by equation (20), and $G_{\mathrm{R}}$ is given by equation (21).

Applying the above equations results in Table 3 and Table 4.

Table 3. The $G_{\mathrm{R}}$-table showing $G_{\mathrm{R}}$ and $2 G_{\mathrm{R}}$ in $\mathrm{dB}$ when $n=16$ and $r=4$.

\begin{tabular}{|c|c|c|}
\hline$R$ & $G_{\mathrm{R}}$ & $2 G_{\mathrm{R}}$ \\
\hline 0 & 0 & 0 \\
\hline 1 & .53 & 1 \\
\hline 2 & 1.02 & 2 \\
\hline 3 & 1.50 & 3 \\
\hline 4 & 1.94 & 4 \\
\hline 5 & 2.36 & 5 \\
\hline 6 & 2.77 & 6 \\
\hline 7 & 3.15 & 6 \\
\hline 8 & 3.52 & 7 \\
\hline 9 & 3.88 & 8 \\
\hline 10 & 4.22 & 8 \\
\hline 11 & 4.54 & 9 \\
\hline 12 & 4.86 & 10 \\
\hline 13 & 5.17 & 10 \\
\hline 14 & 5.46 & 11 \\
\hline 15 & 5.74 & 11 \\
\hline
\end{tabular}

Table 4. The $\left(G_{c}-G_{m}\right)$ table showing in dB's $G_{m},\left(G_{c}-\right.$ $\left.G_{m}\right)$, and $2\left(G_{c}-G_{m}\right)$ when $n=16$ and $r=4$.

\begin{tabular}{|c|c|c|c|}
\hline$m$ & $G_{\mathrm{m}}$ & $G_{\mathrm{c}}-G_{\mathrm{m}}$ & $2\left(G_{\mathrm{c}}-G_{\mathrm{m}}\right)$ \\
\hline 0 & 0 & 96.33 & 193 \\
\hline 1 & 6.02 & 90.31 & 181 \\
\hline 2 & 12.04 & 84.29 & 169 \\
\hline 3 & 18.06 & 78.27 & 157 \\
\hline 4 & 24.08 & 72.25 & 145 \\
\hline 5 & 30.10 & 66.23 & 132 \\
\hline 6 & 36.12 & 60.21 & 120 \\
\hline 7 & 42.14 & 54.19 & 108 \\
\hline 8 & 48.16 & 48.16 & 96 \\
\hline 9 & 54.19 & 42.14 & 84 \\
\hline 10 & 60.21 & 36.12 & 72 \\
\hline 11 & 66.23 & 30.10 & 60 \\
\hline 12 & 72.25 & 24.08 & 48 \\
\hline 13 & 78.27 & 18.06 & 36 \\
\hline 14 & 84.29 & 12.04 & 24 \\
\hline 15 & 90.31 & 6.02 & 12 \\
\hline
\end{tabular}

To illustrate how to use these tables, consider the case where $n=16, r=4$, and

$w=(1010,1010,1100,0011)_{2}$

From equation (4) $m=15$ and from equation (7) $R=5$. The last column of Table 4 shows that $2\left(G_{c}-G_{m}\right)=12$ when $m=15$. In the last column of Table 3 , when $R=5$, it is 
seen that $2 G_{R}=5$. Subtracting these two values gives $-2 G=12-5=7$. Therefore, $G \approx-3.5 \mathrm{~dB}$. The actual value based on equation (2) is $G=-3.517 \mathrm{~dB}$.

\subsection{An assembly language implementation}

An 8051 assembly language implementation of the presented algorithm for the case when $n=16$ and $r=4$ is shown below. The assembly language subroutine is written such that it can be called from an $8051 \mathrm{C}$ program using the Silicon Labs [10] and Keil [11] development tools. The value of $w$ is passed in as a 16-bit unsigned integer using $R 6$ for the most significant byte and $R 7$ for the least significant byte. The $-2 G$ value is returned as an unsigned character in $R 7$.

Table 5. An 8051 assembly language implementation of the refined algorithm when $n=16$ and $r=4$.

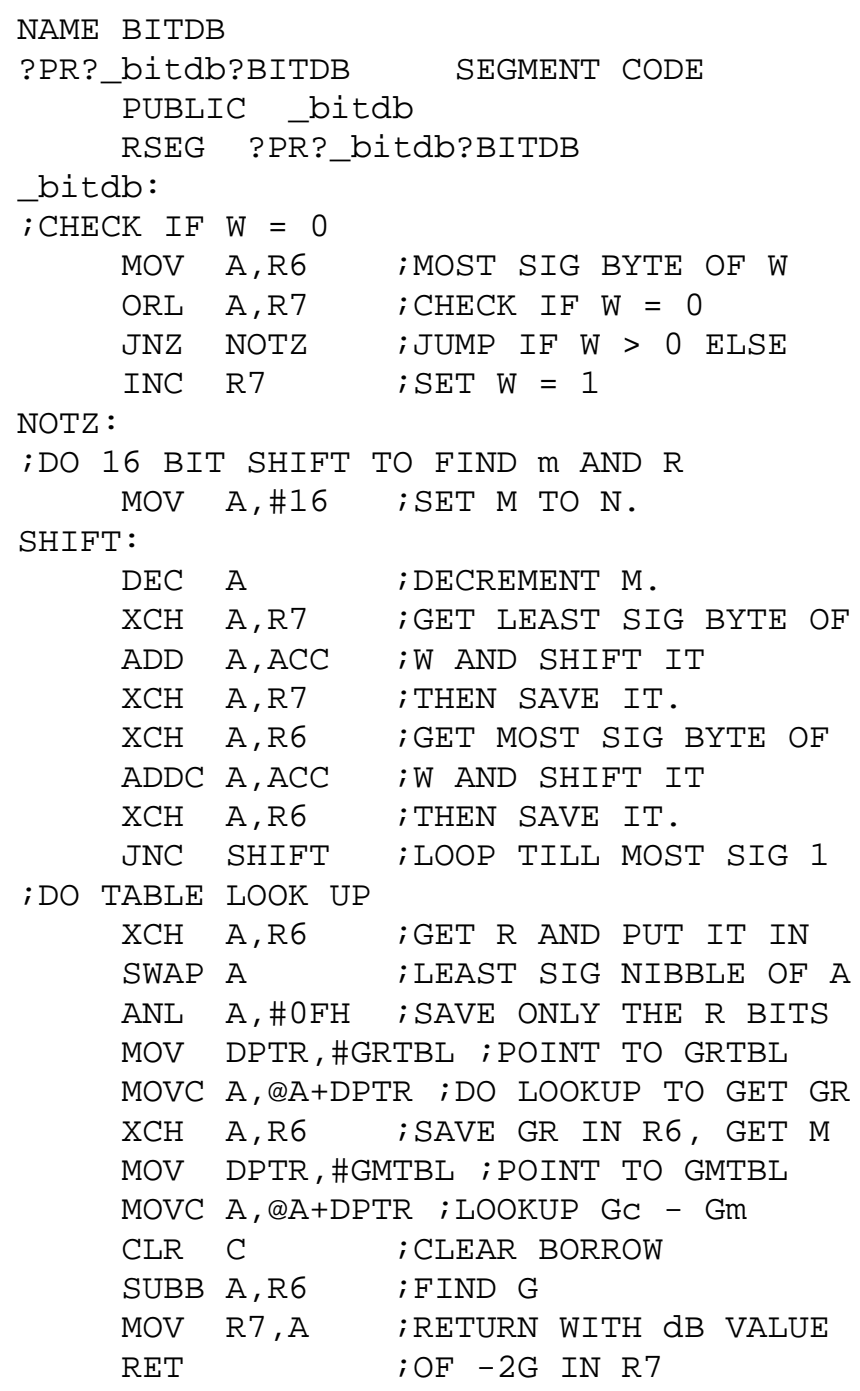

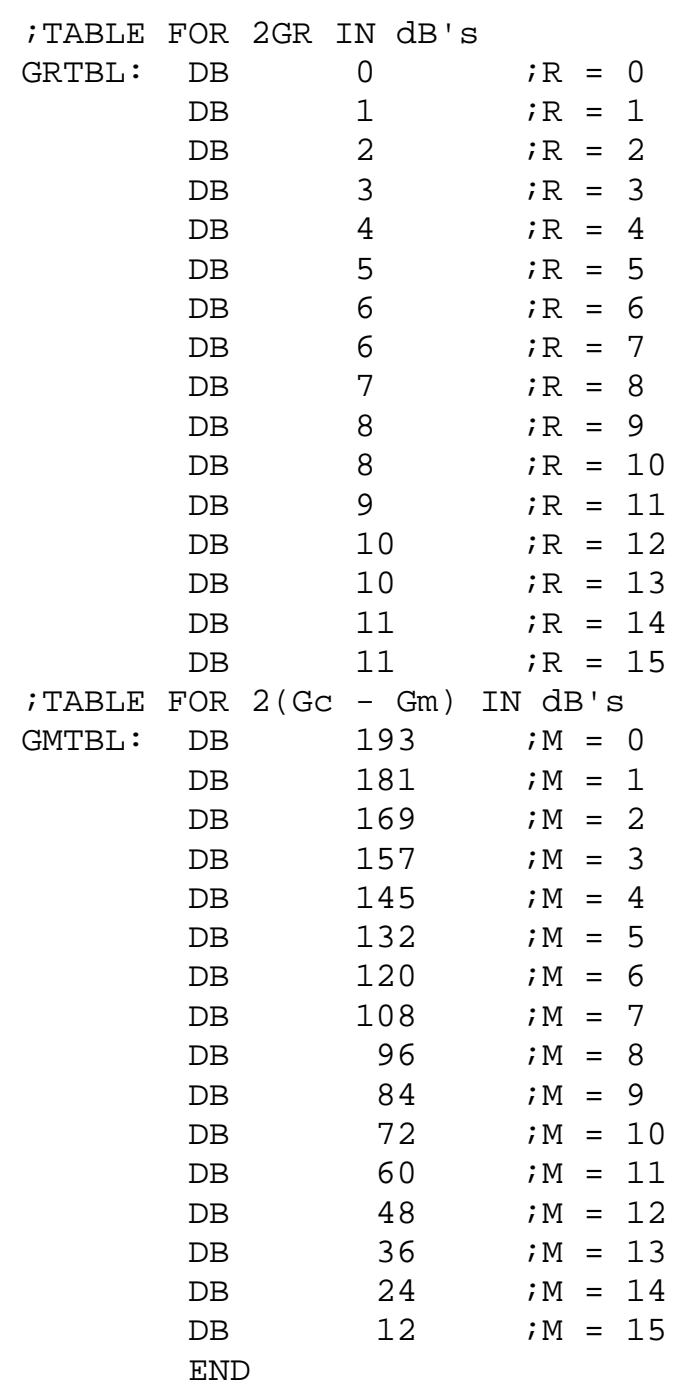

\section{Conclusion}

A technique, that is both fast and easy to implement, has been developed for converting a binary integer, $w$, to its $\mathrm{dB}$ value. The method takes advantage of the fact that in practice decibels do not need to be calculated to high precision. This makes it possible to use two small lookup tables to find the $\mathrm{dB}$ value.

The method has been illustrated for the specific case where $w$ is a 16-bit unsigned integer, and where the four bits following the most significant "1" of $w$ are used to approximate $w$. In this case, using two 16-byte lookup tables, it is possible to find the $\mathrm{dB}$ value of $w$ to within about $1 / 2 \mathrm{~dB}$. An 8051 assembly language program is provided that implements this case. The algorithm could also easily be coded in VHDL and synthesized for implementation in an FPGA. 


\section{References}

[1] Jean-Pierre Deschamps, Gery Jean Antoine Bioul, and Gustavo D. Sutter, Synthesis of Arithmetic Circuits FPGA, ASIC, and Embedded Systems, Wiley-Interscience, Hoboken, New Jersey, 2006.

[2] Milos D. Ercegovac and Tomas Lang, Digital Arithmetic, Morgan Kaufmann, San Francisco, 2004.

[3] Kai Hwang, Computer Arithmetic Principles, Architecture, and Design, John Wiley and Sons, New York, 1979.

[4] J. Koren, Computer Arithmetic Algorithms, Prentice Hall, Englewood Cliffs, NJ, 1993.

[5] D. K. Kostopoulos, “An Algorithm for the Computation of Binary Algorithms," IEEE Trans. On Computers, Vol 40, pp. 1267-1270, November 1991.

[6] Yi Wan and Chin-Long Wey, "Efficient Algorithms for Binary Logarithmic Conversion and Addition," IEEE Proceedings Computers and Digital Techniques, Vol 146, pp. 168-172.

[7] W. M. Humphreys, Q. A. Shams, S. S. Graves, B. S. Sealey, S M. Bartram, T. Comeaux, "Applications of MEMS Microphone Array Technology to Airframe Noise Measurements”, Extended abstract presented at the $11^{\text {th }}$ AIAA/CEAS Aeroacoustics Conference, Monterey, CA. May 23-25, 2005.

[8] Qamar A. Shams, "Design and Fabrication of 128-channel MEMS-based Acoustic Array” presented at Acoustic Society of America, San Diego, CA. November 16, 2004.

[9] Q. A. Shams, S. M. Bartram, W. H. Humphreys, and A. J. Zuckewar. "Phase Calibration of Microphones by Measurements in the Free-field,” Inter-Noise 2006, Honolulu, Hawaii, December 3-6, 2006.

[10] http://www.silabs.com/tgwWebApp/public/index.htm

[11] http://www.keil.com/ 